Journal of Engineering and Applied Sciences 14 (Special Issue 1): 3860-3865, 2019

ISSN: 1816-949X

(C) Medwell Journals, 2019

\title{
Visible Light Communication (VLC) System with Reduced Peak to Average Power Ratio (PAPR) for High Speed Data Transmission
}

\author{
S. Yogeeswaran and G.P. Ramesh \\ Department of ECE, St. Peter's University, Chennai, India
}

\begin{abstract}
In recent times, Visible Light Communication (VLC) is used for high speed transmission using optical wireless communication. VLC contains high power LEDs for high speed data communication. This technique has throughput limitations because the LED bandwidth is limited. To improve the data rate and spectral efficiency of the VLC by using the Orthogonal Frequency Division Multiplexing (OFDM). To propose a novel OFDM based VLC technique improving the efficiency compared to the conventional optical based OFDM system. The existing technique obtained the real and positive values from IFFT operation. The values are taken from the Hermitian symmetry and signal clipping approach, it increased the Peak-to-Average Power Ratio (PAPR). The proposed technique reduces the PAPR without using the Hermitian symmetry. It also increases the efficiency of the system.
\end{abstract}

Key words: Visible Light Communication (VLC), Orthogonal Division Multiplexing (OFDM), Peak-to-Average Power Ratio (PAPR), hermitian symmetry, signal clipping, efficiency

\section{INTRODUCTION}

Visible light communication is a type of data communication use the visible light frequency ranges sbetween $400-800 \mathrm{THz}$ and wavelength range is $780-375 \mathrm{~nm}$. It is type of optical wireless communication technology. It uses LEDs or fluorescent lamp to transmit the data over wireless at the rate of $500 \mathrm{Mbit} / \mathrm{sec}$. Photo diode or photo detectors are used to receive the signals. Today all are using Orthogonal Frequency Division Multiplexing (OFDM) based Visible Light Communication (VLC) for high speed transmission. VLC is a fast and latest wireless communication technologies for optical communication using LEDs. The uses of LED for the communication improve the efficiency of power and reduction of cost. VLC is combined with OFDM for achieving high spectral efficiency and less inter-symbol interferences. Different OFDM like asymmetrically clipped optical OFDM(ACO-OFDM), unipolar OFDM(U-OFDM) are the types to generate the OFDM signal suitable for LED light. Achieve a real valued time domain signal by using Inverse Fast Fourier Transform (IFFT). The changing of signal from frequency to time is reduce the spectral efficiency and increases the complexity of the system. To overcome the drawback and achieve a good bandwidth by using ACO-OFDM. ACO-OFDM ignores or clipping the negative vector, it concentrates only the positive value and the remaining values are set to zero. The results of the ACO-OFDM system is unipolar signal, sacrifices the bandwidth properties. OFDM system involves higher order modulation technique for higher spectral efficiency and BER. Different modulation schemes like QAM, CAP are used to get efficient results. One of the main problems in OFDM is Peak-to-Average Power Ratio (PAPR), PAPR leads to increases the signal distortion and performance degradation. The aim of the OFDM system designer is achieve a high throughput and efficiency without increasing the PAPR parameter. Pilot Symbol Phase Rotation (PSR) technique is used to avoid the high PAPR and higher BER ratio. Signal to Noise Ratio (SNR) also increased by using the PSR technique. Finally, the completed OFDM system is combined with photo diode and photo detector for optical wireless transmission. Li-Fi is the most recent technique for data communication using light. It is more secure than other communication technique (Wi-Fi).

Literature review: Goroshko et al. (2015) proposed the high speed visible light communication using synchronization steps towards reliable data detection to attain high robustness as well as less difficulties. Schmidt-Cox and Park autocorrelation as well as a cross-correlation based approach scheme examined. Finally, optimized preamble structure has been proposed. Preamble boosting and fine synchronization can also be applied to reach a similar performance like optimal cross-correlation at reduced complexity.

Corresponding Author: S. Yogeeswaran, Department of ECE, St. Peter's University, Chennai, India 
Popoola et al. (2015) explained OFDM with non uniform bit loading and VLC system through LED bandwidth to avoid the throughput limitations. Also PAPR along with OFDM signal is reduced by using pilot symbol phase rotation method. This combination of methods attains high throughput without penalties for incurring the high PAPR.

Medina et al. (2016) proposed synchronization scheme on the bases of timing estimation method with the help of new preamble weighted. This method of synchronization scheme is very efficient and performance has been evaluated by simulations and experimentally by means of prototype.

Du et al. (2015) described a novel negative mirror image synchronization algorithm. The sequences of the process has be re designed in terms of the Unipolar Orthogonal Frequency Division Multiplexing (U-OFDM). The performance has been analyzed using simulation results.

Kizilirmak et al. (2015) investigated relay-assisted Visible Light Communication (VLC) system where an intermediate light source cooperates with the main light source. Two light sources: one is the information source employed on the ceiling and the other one is a task light where the task light performs relaying to assist the communication and operates in half-duplex mode for both amplify-and-forward and decode-and-forward relaying is utilized.

Abdulkafi et al. (2017) proposed efficient indoor visible light communication by increasing the data rate and and spectral efficiency. Compare the conventional method proposed method shows significant reduction in PAPR.

Sun et al. (2017) explained three hybrid modulation scheme for VLC based OFDM system are evaluated which are asymmetrically clipped DC-biased optical OFDM, hybrid asymmetrically clipped optical OFDM and layered asymmetrically clipped optical OFDM (LACO-OFDM).

$\mathrm{Xu}$ et al. (2016) proposed asymmetric signal reconstruction, instead of direct clipping, in the time domain before transmission. It refines the time-domain statistics of the Optical OFDM signals. PAPR of the signals is effectively reduced. To recover the received signals precisely, optimal maximum a posteriori detection method has been developed. Simulation result done by proposed method BER performance compared with conventional OOFDMs under both an ideal and a dispersive Visible Light Communication (VLC) channel via. our prototype.

Cailean and Dimian (2017) explained several research directions of VLC. The challenges for VLC usage in vehicle applications addressed by this survey are: increasing the robustness to noise; increasing the communication range; enhancing mobility; performing distance measurements and visible light positioning; increasing data rate; developing parallel VLC and developing heterogeneous dedicated short range communications and VLC nets. Also, proposes several future research directions for the automotive VLC applications.

Gancarz et al. (2013) Advances in Solid-State Lighting (SSL) are enabling LEDs to be the dominant source for future human-made light. The adoption of LEDs is expected to significantly reduce energy consumption and facilitate precise intensity and color control of illuminated spaces. SSL also makes possible the use of Visible Light Communications (VLC) in which data are emitted from luminaires through subtle intensity variations. This study focuses on the impact of lighting requirements on VLC systems, especially as found in indoor deployment scenarios. We also consider dimming control and its impact on color quality in the context of measured vs. perceived light levels.

Vegni and Little (2012) described mobility in VLC devices and propose an approach to managing handover adopted from wireless cellular systems but with very small cells and directional characteristic. Moreover, this technique provides the coordination and cooperation among VLC devices to achieve seamless connectivity in two different indoor scenarios consisting of overlapping (uniform lighting) and non-overlapping (spotlighting) cases.

Chang et al. (2012) proposed a spectrum sensor array to realize the receiver side to achieve less interference. By design the weighting for the individual spectrum sensor SIR output can be Largent. Also signal fusion algorithm has be presented. Simulation results demonstrate that robust interference rejection is possible using the low-cost spectrum sensor array.

Pollet et al. (1995) described the transmission of M-PSK in addition to M-QAM modulated Orthogonal Frequency Division Multiplexed (OFDM) signals over an Additive White Gaussian Noise (AWGN) channel is measured.

Grobe et al. (2013) investigated mobility in VLC devices, also propose an approach to managing handover adopted from wireless cellular systems but with very small cells and directional characteristic. Results indicate how different design decisions in supporting luminaire placement achieve full coverage at varying data rates.

\section{MATERIALS AND METHODS}

Orthogonal Frequency Division Multiplexing (OFDM) system for VLC systems: Orthogonal Frequency Division Multiplexing (OFDM) system is used as the efficient multiplexing scheme as well as the Orthogonal Frequency 

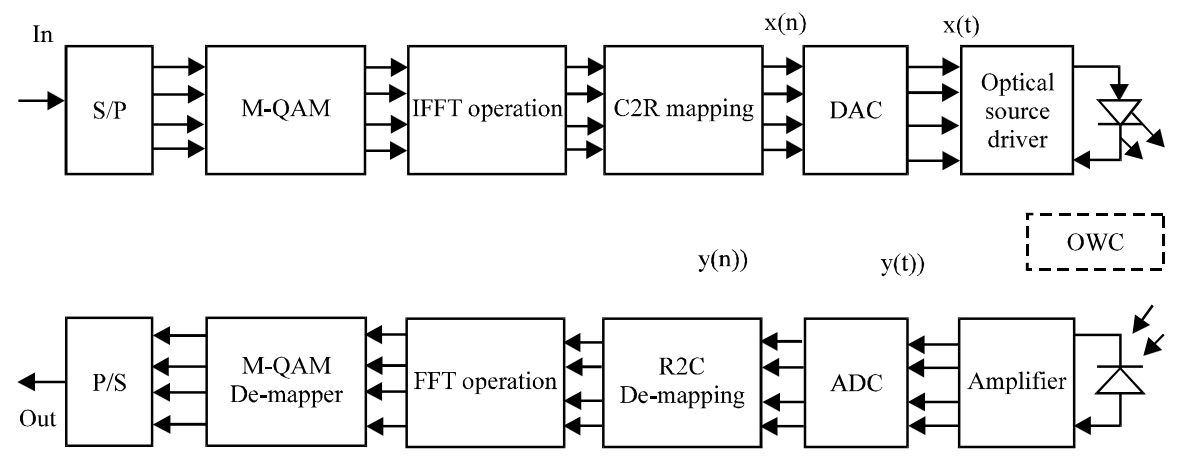

Fig. 1: Architecture for the OFDM based VLC system model

Division Multiplexing (OFDM) is used as the digital modulation technique for the efficient data communication systems. In the Long-term Evaluation (LTE) system, the requirement of the digital modulations is fulfilled by using the two modulation techniques.

That is Single Carrier Frequency Division Multiplexing (SC-FDMA) and Orthogonal Frequency Division Multiplexing (OFDM). SC-FDMA system is precoded system of the OFDM systems with the symbols precoding by using the Discrete Fourier Transform (DFT). Due to the reflections of the structure, the input signal from the base station is forward to the receiver section by using the various paths. The various additions of the signals in the transmitter section are causing the some fluctuations in the receiver area. These amplitude changes are known as the multipath fading. The multipath fading is affecting the performance of the overall system. In the traditional mobile networks, the requirement of the digital modulation is achieved by using the single carrier modulation technique with the equalization of the time domain signal. That single carrier modulation scheme is not suitable for high speed data rate transmission due to the complexity of the modulation scheme. In field of Long-Term Evaluation (LTE) systems, the conventional single carrier modulation scheme is not applicable for high speed data transmission systems. In OFDM systems, the high speed data transmission is highly achieved with the better equalizations. The signal equalization is performed in the frequency domain, so, the complexity of the conventional single carrier modulation scheme is highly reduced by using the OFDM technique. The architecture for OFDM based VLC system model has shown in the Fig. 1. The availability of the Digital Signal Processing (DSP) is one of the main concerns for the implementations of the OFDM systems by using the Fast Fourier Transform (FFT).

Complex to Real (C2R) operation: A Complex to Real (C2R) operation is one of the important process in OFDM based VLC communication system. The real and complex value from the IFFT is coded into a unique real value function. The mathematical expression of $\mathrm{C} 2 \mathrm{R}$ is defined as Eq. 1:

Where:

$$
X_{R}=f\left(x_{c}=f(p, a, b)\right)
$$

$\mathrm{x}_{\mathrm{c}}=$ The complex signal generated from IFFT operation, it can be expressed as $\mathrm{a}+\mathrm{jb}$

' $a$ ' $=$ The real part

' $\mathrm{b}$ ' = The imaginary part of the function

Funtion ' $\mathrm{f}$ ' = Converts the complex valued number into real valued number

Time domain complex numbers is encoded into frequency domain with same integers. The mapping function is enough for VLC systems. The real valued OFDM signal is characterized by high Peak-to-Average Power Ratio (PAPR). It can be represented as Eq. 2:

$$
\operatorname{PAPR}=\frac{\max \left(\mathrm{x}_{\mathrm{R}}\right)^{2}}{\mathrm{E}\left[\mathrm{x}_{\mathrm{R}}^{2}\right]}
$$

Where:

$\max \left(\mathrm{x}_{\mathrm{R}}^{2}\right)=$ Maximum value of OFDM signal

$\mathrm{E}\left(\mathrm{x}_{\mathrm{R}}^{2}\right)=$ Mean of those values

Visible Light Communication (VLC) system features: Visible Light Communication (VLC) systems is providing the some features for the efficient data transmission systems. That features are explained below.

Power efficiency: The power utilizations are achieved by using the indoor lights for the purpose of the lightning and also the data communications is also performed by using the indoor lights to consume the energy. For Visible Light Communication (VLC) systems, there is no requirement for the additional energy resources.

Security for information: In VLC systems, the data communication is performed only within the room or 
particular area due to the availability of the visible light systems. Communicated data is to be secured highly compared to the RF wireless systems.

Efficient interference: The Visible Light Communication (VLC) systems utilize the visible light for efficient data transmission, so, the system is provides the zero interference for the hospitals and RF wireless not applicable systems.

Beam routing: Visible light is easily able to direct to the systems, so, the routing of the expensive equipment is not required. The visible light systems is offers the low expensive optical equipments.

System performance: To implement the Orthogonal Frequency Division Multiplexing (OFDM) Systems in the continuous time domain requires the various number of filters and modulators, leads the complexity of the overall system performance. However, the requirement of various elements at both the transmitter and receiver is reduced by using the Inverse Fast Fourier Transform (IFFT) in the design of OFDM systems. Mathematically Eq. 3:

$$
\operatorname{sg}(t)=\sum_{k=0}^{N-1} d 0, k^{j 2 \pi n k t}
$$

where, $\mathrm{t}=\mathrm{mT}_{\mathrm{b}} \mathrm{N}$ Eq. 4 :

$$
\begin{gathered}
\operatorname{sg}(\mathrm{t})=\sum_{\mathrm{k}=0}^{\mathrm{N}-1} \mathrm{~d} 0, \mathrm{kmT}_{\mathrm{b}} / \mathrm{N} \\
\mathrm{y}(\mathrm{m})=\mathrm{s}(\mathrm{t}) \mid \mathrm{t}=\frac{\mathrm{mT}_{\mathrm{b}}}{\mathrm{n}}=\sum_{\mathrm{k}=0}^{\mathrm{N}-1} \mathrm{~d} 0, \mathrm{ke}^{\frac{2 \pi \mathrm{km}}{\mathrm{N}}}
\end{gathered}
$$

By using the FFT/IFFT for the implementations of the OFDM systems, the efficient number of elements simplifications are achieved compare to the modulation and demodulation using modulators. The FFT and IFFT are mainly used for the efficient data transformations from the time domain into frequency domain and frequency domain into time domain. These transformations are highly used for the efficient data transmission systems. Then the output of the OFDM system is generated by the performing the IFFT of the given samples where $\mathrm{N}=\mathrm{QM}$. At the receiver side of the IFFT block is transmitted by the user ' $i$ ' is given by Eq. 6:

$$
X^{I}(N)=\frac{1}{N} \sum_{k=0}^{N-1} X^{I[K] e^{j 2-\left(\frac{k}{N}\right)^{n}}}
$$

In an OFDM based data transmission systems, the data sending based on the form of blocks. By using the Inverse Fast Fourier Transform (IFFT), the signal is provide in terms of the time domain and also the output signal samples are equal to the sub carrier modulation samples. However, the OFDM system is used by the Q users and the each user signal is allocated to the particular $\mathrm{M}$ number of sub carriers samples at the given time sequence.

\section{RESULTS AND DISCUSSION}

Performance analysis: In this chapter, the performance analysis of the Visible Light Communication (VLC) system based OFDM technique is evaluated by using modelsim XE (Fig. 2). Simulation results for the OFDM based VLC

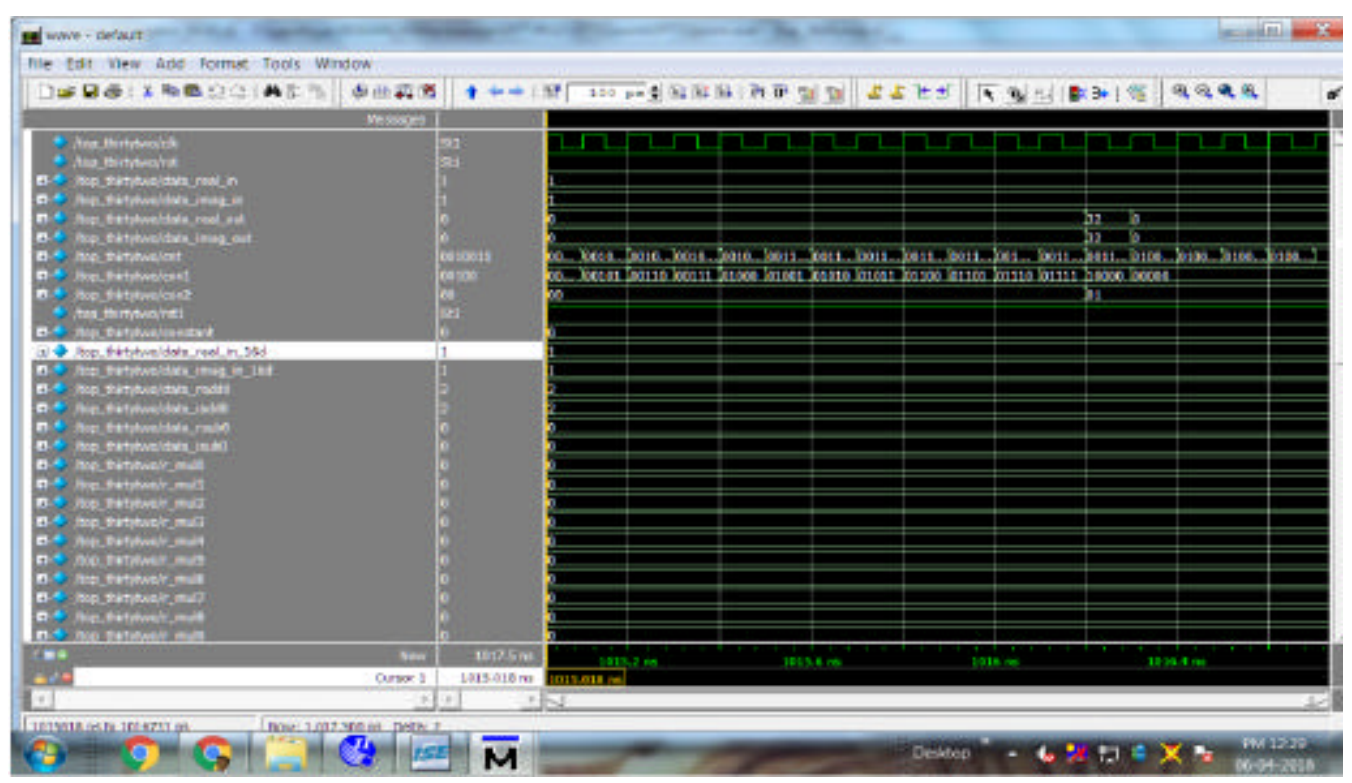

Fig. 2: Simulation output for OFDM system with FFT/IFFT 


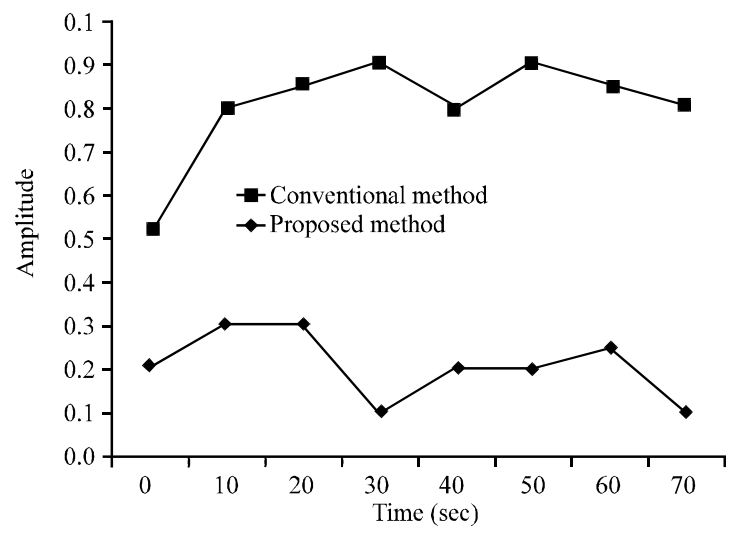

Fig. 3: Comparison between output signals strength of conventional and proposed schemes

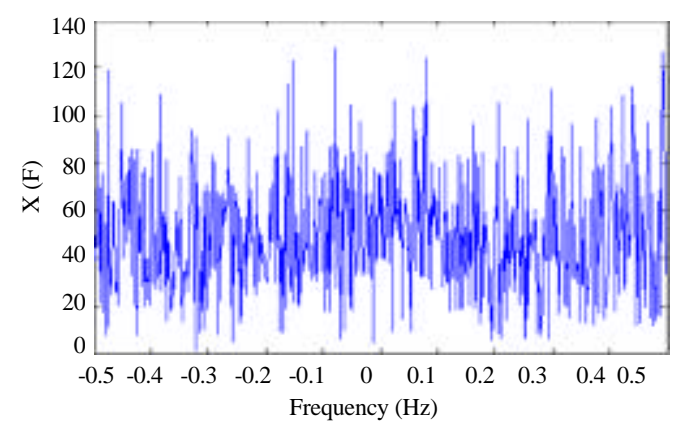

Fig. 4: Signal spectrum of the OFDM signal

system are used to evaluate the performance of the system and the Peak-Average Power Ratio (PAPR) is analyzed and the simulated output signal of VLSI and MATLAB is illustrated in Fig. 2 and 4. Figure 2 shows the output of FFT process in the OFDM system. The input given to the FFT is time based signal, it convert time signal into frequency signal. The real and imaginary input bits are given to simulator; it generates output based on the butterfly diagram. Since, the modulation of the OFDM is achieved by using the novel modulation technique with the efficient Bit-Error-Rate (BER). In the proposed method, the modulation techniques with various sub-carriers are used for the efficient BER analysis. The real positive signals are determined by applying the signal samples to the Inverse Fast Fourier Transform (IFFT).

Figure 3 shows the signal strength of the OFDM signal is higher when compared to the conventional method. The signal is changing according to the time.

Figure 4 shows that the signal spectrum of the OFDM signal. The spectrum has higher frequency. Figure 5 shows the comparison of BER between the conventional and proposed method. Proposed method has less BER than compared to the conventional method.

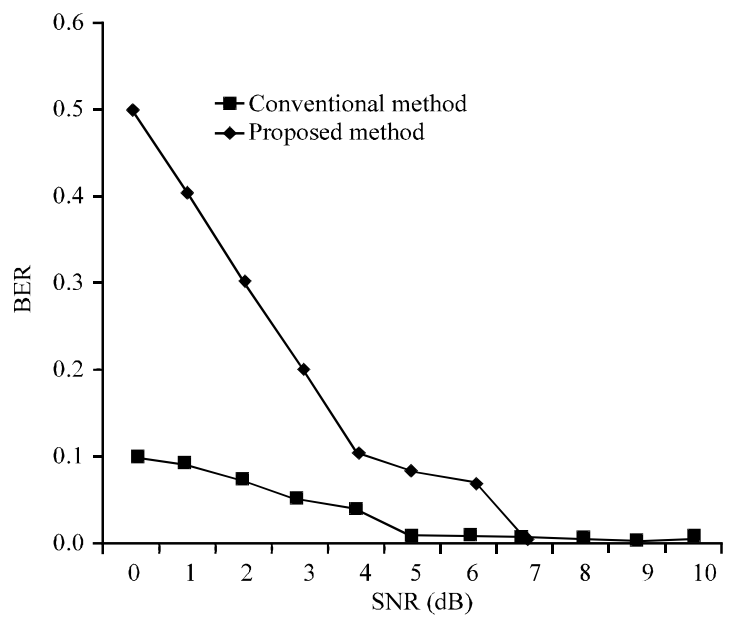

Fig. 5: Comparison of Bit-Error-Rate (BER) between conventional and proposed scheme

\section{CONCLUSION}

In this research, the Orthogonal Frequency Division Multiplexing (OFDM) system for high speed data transmission is analyzed and implemented in terms of the VLSI design environment. The PAPR reduction is achieved by using the OFDM systems for data communication systems with the efficient FFT/IFFT system and efficient digital modulation technique. FFT/IFFT transformation is mainly used to perform the both time and frequency domain of the given input samples. OFDM system is one of the most efficient techniques for the wired and wireless data communication systems due to the high data rate and spectral efficiency. However, the Peak-Average Power Ratio (PAPR) improvisation is one of the main concerns in the data communication systems. The improvement of the PAPR is also causes the some changes in the Bit-Error-Rate (BER) and Signal-Noise Ratio (SNR) of the systems. These analyses are evaluated by using the MATLAB and the performance of the VLC system based OFDM with the FFT/IFFT systems are implemented by using the modelsim XE.

\section{REFERENCES}

Abdulkafi, A.A., M.Y. Alias and Y.S. Hussein, 2017. A novel approach for PAPR reduction in OFDM-based visible light communications. Proceedings of the 2017 International Conference on Platform Technology and Service (PlatCon'17), February 13-15, 2017, IEEE, Busan, South Korea, ISBN:978-1-5090-5141-0, pp: $1-4$. 
Cailean, A. and M. Dimian, 2017. Current challenges for visible light communications usage in vehicle applications: A survey. IEEE. Commun. Surv. Tutorials, 99: 1-1.

Chang, C.C., Y.J. Su, U. Kurokawa and B.I. Choi, 2012. Interference rejection using filter-based sensor array in VLC systems. IEEE. Sens. J., 12: 1025-1032.

Du, Z., X. Zhou and K. Long, 2015. A novel timing synchronization method for U-OFDM-based visible light communication. Proceedings of the 24th Conference on Wireless and Optical Communication (WOCC'15), October 23-24, 2015, IEEE, Taipei, Taiwan, ISBN:978-1-4799-8849-5, pp: 63-66.

Gancarz, J., H. Elgala and T.D. Little, 2013. Impact of lighting requirements on VLC systems. IEEE. Commun. Mag., 51: 34-41.

Goroshko, K., K. Manolakis, L. Grobe and V. Jungnickel, 2015. Low-latency synchronization for OFDM-based visible light communication. Proceedings of the 2015 IEEE International Conference on Communication Workshop (ICCW'15), June 8-12, 2015, IEEE, London, UK., ISBN:978-1-4673-6305-1, pp: 1327-1332.

Grobe, L., A. Paraskevopoulos, J. Hilt, D. Schulz and F. Lassak et al., 2013. High-speed visible light communication systems. IEEE. Commun. Mag., 51: 60-66.

Kizilirmak, R.C., O. Narmanlioglu and M. Uysal, 2015. Relay-assisted OFDM-based visible light communications. IEEE. Trans. Commun., 63: $3765-3778$.
Medina, M.F.G., O. Gonzalez, S. Rodriguez and I.R. Martin, 2016. Timing synchronization for OFDM-based visible light communication system. Proceedings of the 2016 Symposium on Wireless Telecommunications (WTS'16), April 18-20, 2016, IEEE, London, UK., ISBN:978-1-5090-0314-3, pp: 1-4.

Pollet, T., M. van Bladel and M. Moeneclaey, 1995. Ber sensitivity of OFDM systems to carrier frequency offset and wiener phase noise. IEEE Commun., Trans., 43: 191-193.

Popoola, W.O., Z. Ghassemlooy and B.G. Stewart, 2015. Optimising OFDM based visible light communication for high throughput and reduced PAPR. Proceedings of the 2015 IEEE International Conference on Communication Workshop (ICCW'15), June 8-12, 2015, IEEE, London, UK., ISBN:978-1-4673-6305-1, pp: 1322-1326.

Sun, Y., F. Yang and J. Gao, 2017. Comparison of hybrid optical modulation schemes for visible light communication. IEEE. Photonics J., 9: 1-13.

Vegni, A.M. and T.D. Little, 2012. Handover in VLC systems with cooperating mobile devices. Proceedings of the 2012 International Conference on Computing Networking and Communications (ICNC'12), January 30-February 2, 2012, IEEE, Maui, Hawaii, ISBN:978-1-4673-0008-7, pp: 126-130.

Xu, J., W. Xu, H. Zhang and X. You, 2016. Asymmetrically reconstructed optical OFDM for visible light communications. IEEE. Photonics J., 8: 1-18. 